\title{
Fractional Hadamard and Fejér-Hadamard inequalities for exponentially $m$-convex function
}

\author{
Sajid Mehmood and Ghulam Farid
}

\begin{abstract}
Fractional integral operators play a vital role in the advancement of mathematical inequalities. The aim of this paper is to present the Hadamard and the Fejér-Hadamard inequalities for generalized fractional integral operators containing Mittag-Leffler function. Exponentially $m$-convexity is utilized to establish these inequalities. By fixing parameters involved in the Mittag-Leffler function Hadamard and the Fejér-Hadamard inequalities for various well known fractional integral operators can be obtained.
\end{abstract}

Mathematics Subject Classification (2010): 26B25, 26A33, 26A51, 33E12.

Keywords: Convex functions, exponentially $m$-convex functions, Hadamard inequality, Fejér-Hadamard inequality, fractional integral operators, Mittag-Leffler function.

\section{Introduction}

A function $f:[a, b] \rightarrow \mathbb{R}$ is said to be convex, if for all $x, y \in[a, b]$ and $z \in[0,1]$, the following inequality holds:

$$
f(z x+(1-z) y) \leq z f(x)+(1-z) f(y) .
$$

If inequality (1.1) is reversed, then $f$ is said to be concave.

Convex functions are equivalently defined by well known Hadamard inequality stated as follows:

Theorem 1.1. Let $f:[a, b] \rightarrow \mathbb{R}$ be a convex function such that $a<b$. Then the following inequality holds:

$$
f\left(\frac{a+b}{2}\right) \leq \frac{1}{b-a} \int_{a}^{b} f(x) d x \leq \frac{f(a)+f(b)}{2} .
$$

Fejér-Hadamard inequality is a weighted version of the Hadamard inequality established by Fejér [13]. 
Theorem 1.2. Let $f:[a, b] \rightarrow \mathbb{R}$ be a convex function and $g:[a, b] \rightarrow \mathbb{R}$ be a nonnegative, integrable and symmetric to $\frac{a+b}{2}$. Then the following inequality holds:

$$
f\left(\frac{a+b}{2}\right) \int_{a}^{b} g(x) d x \leq \int_{a}^{b} f(x) g(x) d x \leq \frac{f(a)+f(b)}{2} \int_{a}^{b} g(x) d x .
$$

Many researchers are continuously working on inequalities (1.2) and (1.3), and have produced very interesting results for convex and related functions (for example see, $[1,5,6,7,8,9,12,11,14,21,22])$.

Next we define exponentially convex function.

Definition 1.3. $[4,7]$ A function $f:[a, b] \rightarrow \mathbb{R}$ is said to be exponentially convex, if for all $x, y \in[a, b]$ and $z \in[0,1]$, the following inequality holds:

$$
e^{f(z x+(1-z) y)} \leq z e^{f(x)}+(1-z) e^{f(y)} .
$$

The concept of exponentially $m$-convex functions was introduced by Rashid et al. in [18]. It is defined as follows:

Definition 1.4. A function $f:[a, b] \rightarrow \mathbb{R}$ is said to be exponentially $m$-convex, where $m \in(0,1]$, if for all $x, y \in[a, b]$ and $z \in[0,1]$, the following inequality holds:

$$
e^{f(z x+m(1-z) y)} \leq z e^{f(x)}+m(1-z) e^{f(y)} .
$$

Remark 1.5. If we take $m=1$ in (1.5), then (1.4) is achieved.

Mittag-Leffler function was introduced by the Swedish mathematician [15]. It is defined as follows:

$$
E_{\sigma}(t)=\sum_{n=0}^{\infty} \frac{t^{n}}{\Gamma(\sigma n+1)},
$$

where $\Gamma($.$) is the gamma function and t, \sigma \in \mathbb{C}, \Re(\sigma)>0$.

In the solution of kinetic equations and fractional differential equations the MittagLeffler function arises naturally. It is generalized by many researchers due to it's importance. Recently in [3], Andrić et al. introduced generalized Mittag-Leffler function defined as follows:

Definition 1.6. [3] Let $\mu, \sigma, l, \rho, c \in \mathbb{C}, \Re(\mu), \Re(\sigma), \Re(l)>0, \Re(c)>\Re(\rho)>0$ with $p \geq 0, r>0$ and $0<q \leq r+\Re(\mu)$. Then the extended generalized Mittag-Leffler function $E_{\mu, \sigma, l}^{\rho, r, q, c}(t ; p)$ is defined by:

$$
E_{\mu, \sigma, l}^{\rho, r, q, c}(t ; p)=\sum_{n=0}^{\infty} \frac{\beta_{p}(\rho+n q, c-\rho)}{\beta(\rho, c-\rho)} \frac{(c)_{n q}}{\Gamma(\mu n+\sigma)} \frac{t^{n}}{(l)_{n r}}
$$

where $\beta_{p}$ is the generalized beta function defined by:

$$
\beta_{p}(x, y)=\int_{0}^{1} t^{x-1}(1-t)^{y-1} e^{-\frac{p}{t(1-t)}} d t
$$

and $(c)_{n q}$ is the Pochhammer symbol defined as $(c)_{n q}=\frac{\Gamma(c+n q)}{\Gamma(c)}$. 
Remark 1.7. (1.6) is a generalization of the following Mittag-Leffler functions defined by many authors:

(i) taking $p=0$, it reduces to the Salim-Faraj function $E_{\mu, \sigma, l}^{\rho, r, q}(t)$ defined in [20],

(ii) taking $l=r=1$, it reduces the function $E_{\mu, \sigma}^{\rho, q, c}(t ; p)$ defined by Rahman et al. in [17],

(iii) taking $p=0$ and $l=r=1$, it reduces to the Shukla-Prajapati function $E_{\mu, \sigma}^{\rho, q}(t)$ defined in [23] see also [24],

(iv) taking $p=0$ and $l=r=q=1$, it reduces to the Prabhakar function $E_{\mu, \sigma}^{\rho}(t)$ defined in $[16]$.

The left-sided and right-sided generalized fractional integral operators containing Mittag-Leffler function (1.6) are defined as follows:

Definition 1.8. [3] Let $\omega, \mu, \sigma, l, \rho, c \in \mathbb{C}, \Re(\mu), \Re(\sigma), \Re(l)>0, \Re(c)>\Re(\rho)>0$ with $p \geq 0, r>0$ and $0<q \leq r+\Re(\mu)$. Let $f \in L_{1}[a, b]$ and $x \in[a, b]$. Then the generalized fractional integral operators $\epsilon_{\mu, \sigma, l, \omega, a^{+}}^{\rho, r, q, c} f$ and $\epsilon_{\mu, \sigma, l, \omega, b^{-}}^{\rho, r, q, c} f$ are defined by:

$$
\left(\epsilon_{\mu, \sigma, l, \omega, a^{+}}^{\rho, r, q, c} f\right)(x ; p)=\int_{a}^{x}(x-t)^{\sigma-1} E_{\mu, \sigma, l}^{\rho, r, q, c}\left(\omega(x-t)^{\mu} ; p\right) f(t) d t
$$

and

$$
\left(\epsilon_{\mu, \sigma, l, \omega, b^{-}}^{\rho, r, q, c} f\right)(x ; p)=\int_{x}^{b}(t-x)^{\sigma-1} E_{\mu, \sigma, l}^{\rho, r, q, c}\left(\omega(t-x)^{\mu} ; p\right) f(t) d t .
$$

Remark 1.9. (1.7) and (1.8) are the generalization of the following fractional integral operators defined by many authors:

(i) taking $p=0$, it reduces to the fractional integral operators defined by Salim-Faraj in $[20]$,

(ii) taking $l=r=1$, it reduces to the fractional integral operators defined by Rahman et al. in [17],

(iii) taking $p=0$ and $l=r=1$, it reduces to the fractional integral operators defined by Srivastava-Tomovski in [24],

(iv) taking $p=0$ and $l=r=q=1$, it reduces to the fractional integral operators defined by Prabhakar in [16],

(v) taking $p=\omega=0$, it reduces to the right-sided and left-sided Riemann-Liouville fractional integrals.

As shown in [2] also [10], for the constant function we have:

$$
\left(\epsilon_{\mu, \sigma, l, \omega, a^{+}}^{\rho, r, q, c} 1\right)(x ; p)=(x-a)^{\sigma} E_{\mu, \sigma+1, l}^{\rho, r, q, c}\left(\omega(x-a)^{\mu} ; p\right):=G_{\sigma, \omega, a^{+}}(x ; p),
$$

and

$$
\left(\epsilon_{\mu, \sigma, l, \omega, b^{-}}^{\rho, r, q, c} 1\right)(x ; p)=(b-x)^{\sigma} E_{\mu, \sigma+1, l}^{\rho, r, q, c}\left(\omega(b-x)^{\mu} ; p\right):=G_{\sigma, \omega, b^{-}}(x ; p),
$$

which we use in our results.

In the upcoming section, first we prove the Hadamard inequality for exponentially $m$-convex functions via fractional integral operators defined in (1.7) and (1.8). Also, the Fejér-Hadamard inequality for these operators is obtained. We mention results for particular fractional integral operators associated with (1.7) and (1.8). 


\section{Fractional Hadamard and Fejér-Hadamard inequalities for generalized fractional integral operators}

First we give the fractional Hadamard inequality for exponentially $m$-convex functions via generalized fractional integral operators.

Theorem 2.1. Let $\omega, \mu, \sigma, l, \rho, c \in \mathbb{C}, \Re(\mu), \Re(\sigma), \Re(l)>0, \Re(c)>\Re(\rho)>0$ with $p \geq 0, r>0$ and $0<q \leq r+\Re(\mu)$. Let $f:[a, m b] \subset \mathbb{R} \rightarrow \mathbb{R}$ be a function such that $f \in L_{1}[a, m b]$ with $a<m b$. If $f$ is exponentially $m$-convex function, then the following inequalities hold:

$$
\begin{aligned}
& e^{f\left(\frac{a+m b}{2}\right)} G_{\sigma, \bar{\omega}, a^{+}}(m b ; p) \\
& \leq \frac{\left(\epsilon_{\mu, \sigma, l, \bar{\omega}, a^{+}}^{\rho, r, q,} e^{f}\right)(m b ; p)+m^{\sigma+1}\left(\epsilon_{\mu, \sigma, l, \bar{\omega} m^{\mu}, b^{-}}^{\rho, r, q,} e^{f}\right)\left(\frac{a}{m} ; p\right)}{2} \\
& \leq \frac{m^{\sigma+1}}{2(m b-a)}\left[\left(e^{f(a)}-m^{2} e^{f\left(\frac{a}{m^{2}}\right)}\right) G_{\sigma+1, \bar{\omega} m^{\mu}, b^{-}}\left(\frac{a}{m} ; p\right)\right. \\
& \left.+(m b-a)\left(e^{f(b)}+m e^{f\left(\frac{a}{m^{2}}\right)}\right) G_{\sigma, \bar{\omega} m^{\mu}, b^{-}}\left(\frac{a}{m} ; p\right)\right]
\end{aligned}
$$

where $m \in(0,1]$ and

$$
\bar{\omega}=\frac{\omega}{(m b-a)^{\mu}} .
$$

Proof. Since $f$ is exponentially $m$-convex, we have

$$
e^{f\left(\frac{x+m y}{2}\right)} \leq \frac{e^{f(x)}+m e^{f(y)}}{2} \quad \forall x, y \in[a, m b] \text { and } m \in(0,1] \text {. }
$$

Putting $x=z a+m(1-z) b$ and $y=(1-z) \frac{a}{m}+z b$ in (2.3), we get

$$
2 e^{f\left(\frac{a+m b}{2}\right)} \leq e^{f(z a+m(1-z) b)}+m e^{f\left((1-z) \frac{a}{m}+z b\right)} .
$$

Also from exponentially $m$-convexity of $f$, we have

$$
\begin{aligned}
& e^{f(z a+m(1-z) b)}+m e^{f\left((1-z) \frac{a}{m}+z b\right)} \\
& \leq z e^{f(a)}+m(1-z) e^{f(b)}+m\left(m(1-z) e^{f\left(\frac{a}{m^{2}}\right)}+z e^{f(b)}\right) \\
& =z\left(e^{f(a)}-m^{2} e^{f\left(\frac{a}{m^{2}}\right)}\right)+m\left(e^{f(b)}+m e^{f\left(\frac{a}{m^{2}}\right)}\right) .
\end{aligned}
$$

Multiplying both sides of $(2.4)$ with $z^{\sigma-1} E_{\mu, \sigma, l}^{\rho, r, q, c}\left(\omega z^{\mu} ; p\right)$ and integrating over $[0,1]$, we have

$$
\begin{aligned}
& 2 e^{f\left(\frac{a+m b}{2}\right)} \int_{0}^{1} z^{\sigma-1} E_{\mu, \sigma, l}^{\rho, r, q, c}\left(\omega z^{\mu} ; p\right) d z \\
& \leq \int_{0}^{1} z^{\sigma-1} E_{\mu, \sigma, l}^{\rho, r, q, c}\left(\omega z^{\mu} ; p\right) e^{f(z a+m(1-z) b)} d z \\
& +m \int_{0}^{1} z^{\sigma-1} E_{\mu, \sigma, l}^{\rho, r, q, c}\left(\omega z^{\mu} ; p\right) e^{f\left((1-z) \frac{a}{m}+z b\right)} d z .
\end{aligned}
$$


Putting $u=z a+m(1-z) b$ and $v=(1-z) \frac{a}{m}+z b$ in (2.6), we get

$$
\begin{aligned}
& 2 e^{f\left(\frac{a+m b}{2}\right)} \int_{a}^{m b}(m b-u)^{\sigma-1} E_{\mu, \sigma, l}^{\rho, r, q, c}\left(\bar{\omega}(m b-u)^{\mu} ; p\right) d u \\
& \leq \int_{a}^{m b}(m b-u)^{\sigma-1} E_{\mu, \sigma, l}^{\rho, r, q, c}\left(\bar{\omega}(m b-u)^{\mu} ; p\right) e^{f(u)} d u \\
& +m^{\sigma+1} \int_{\frac{a}{m}}^{b}\left(v-\frac{a}{m}\right)^{\sigma-1} E_{\mu, \sigma, l}^{\rho, r, q, c}\left(m^{\mu} \bar{\omega}\left(v-\frac{a}{m}\right)^{\mu} ; p\right) e^{f(v)} d v .
\end{aligned}
$$

By using (1.7), (1.8) and (1.9), first inequality of (2.1) is achieved.

Now multiplying both sides of $(2.5)$ with $z^{\sigma-1} E_{\mu, \sigma, l}^{\rho, r, q, c}\left(\omega z^{\mu} ; p\right)$ and integrating over $[0,1]$, we have

$$
\begin{aligned}
& \int_{0}^{1} z^{\sigma-1} E_{\mu, \sigma, l}^{\rho, r, q, c}\left(\omega z^{\mu} ; p\right) e^{f(z a+m(1-z) b)} d z \\
& +m \int_{0}^{1} z^{\sigma-1} E_{\mu, \sigma, l}^{\rho, r, q, c}\left(\omega z^{\mu} ; p\right) e^{f\left((1-z) \frac{a}{m}+z b\right)} d z \\
& \leq\left(e^{f(a)}-m^{2} e^{f\left(\frac{a}{m^{2}}\right)}\right) \int_{0}^{1} z^{\sigma} E_{\mu, \sigma, l}^{\rho, r, q, c}\left(\omega z^{\mu} ; p\right) d z \\
& +m\left(e^{f(b)}+m e^{f\left(\frac{a}{m^{2}}\right)}\right) \int_{0}^{1} z^{\sigma-1} E_{\mu, \sigma, l}^{\rho, r, q, c}\left(\omega z^{\mu} ; p\right) d z .
\end{aligned}
$$

Putting $u=z a+m(1-z) b$ and $v=(1-z) \frac{a}{m}+z b$ in $(2.7)$, we get

$$
\begin{aligned}
& \int_{a}^{m b}(m b-u)^{\sigma-1} E_{\mu, \sigma, l}^{\rho, r, q, c}\left(\bar{\omega}(m b-u)^{\mu} ; p\right) e^{f(u)} d u \\
& +m^{\sigma+1} \int_{\frac{a}{m}}^{b}\left(v-\frac{a}{m}\right)^{\sigma-1} E_{\mu, \sigma, l}^{\rho, r, q, c}\left(m^{\mu} \bar{\omega}\left(v-\frac{a}{m}\right)^{\mu} ; p\right) e^{f(v)} d v \\
& \leq \frac{m^{\sigma+1}}{(m b-a)}\left[\left(e^{f(a)}-m^{2} e^{f\left(\frac{a}{m^{2}}\right)}\right) \int_{\frac{a}{m}}^{b}\left(v-\frac{a}{m}\right)^{\sigma} E_{\mu, \sigma, l}^{\rho, r, q, c}\left(m^{\mu} \bar{\omega}\left(v-\frac{a}{m}\right)^{\mu} ; p\right) d v\right. \\
& \left.+(m b-a)\left(e^{f(b)}+m e^{f\left(\frac{a}{m^{2}}\right)}\right) \int_{\frac{a}{m}}^{b}\left(v-\frac{a}{m}\right)^{\sigma-1} E_{\mu, \sigma, l}^{\rho, r, q, c}\left(m^{\mu} \bar{\omega}\left(v-\frac{a}{m}\right)^{\mu} ; p\right) d v\right] .
\end{aligned}
$$

By using (1.7), (1.8) and (1.10), second inequality of (2.1) is achieved.

Corollary 2.2. Suppose that assumptions of Theorem 2.1 hold and let $m=1$. Then following inequalities for exponentially convex function hold:

$$
\begin{aligned}
e^{f\left(\frac{a+b}{2}\right)} G_{\sigma, \omega^{*}, a^{+}}(b ; p) & \leq \frac{\left(\epsilon_{\mu, \sigma, l, \omega^{*}, a^{+}}^{\rho, r, q, c} e^{f}\right)(b ; p)+\left(\epsilon_{\mu, \sigma, l, \omega^{*}, b^{-}}^{\rho, r, q, c} e^{f}\right)(a ; p)}{2} \\
& \leq \frac{e^{f(a)}+e^{f(b)}}{2} G_{\sigma, \omega^{*}, b^{-}}(a ; p)
\end{aligned}
$$

where

$$
\omega^{*}=\frac{\omega}{(b-a)^{\mu}}
$$


In [19], S. Rashid et. al. prove the following Hadamard inequality for exponentially $m$-convex function which has several misprints.

Theorem 2.3. Let $f:[a, m b] \subset \mathbb{R} \rightarrow \mathbb{R}$ be a function such that $f \in L_{1}[a, m b]$ with $a<m b$. If $f$ is exponentially $m$-convex function, then the following inequalities hold:

$$
\begin{aligned}
& 2 e^{f\left(\frac{a+m b}{2}\right)} G_{\sigma,\left(\frac{a+m b}{2}\right)^{+}}(m b ; p) \\
& \leq\left(\epsilon_{\mu, \sigma, q, c}^{\rho, r, \bar{\omega} 2^{\mu},\left(\frac{a+m b}{2}\right)^{+}} e^{f}\right)(m b ; p)+m^{\sigma+1}\left(\epsilon_{\mu, \sigma, l, \bar{\omega} 2^{\mu},\left(\frac{a+m b}{2 m}\right)^{-, r, c}} e^{f}\right)\left(\frac{a}{m} ; p\right) \\
& \leq \frac{a}{(m b-a)}\left(e^{f(a)}-m^{2} e^{f\left(\frac{a}{m^{2}}\right)}\right) G_{\sigma+1,\left(\frac{a+m b}{2}\right)^{+}}(m b ; p) \\
& +m^{\sigma+1}\left(e^{f(b)}+m e^{f\left(\frac{a}{m^{2}}\right)}\right) G_{\sigma,\left(\frac{a+m b}{2 m}\right)^{-}}
\end{aligned}
$$
theorem.

The correct form of the above theorem is stated and proved in the following

Theorem 2.4. Let $\omega, \mu, \sigma, l, \rho, c \in \mathbb{C}, \Re(\mu), \Re(\sigma), \Re(l)>0, \Re(c)>\Re(\rho)>0$ with $p \geq 0, r>0$ and $0<q \leq r+\Re(\mu)$. Let $f:[a, m b] \subset \mathbb{R} \rightarrow \mathbb{R}$ be a function such that $f \in L_{1}[a, m b]$ with $a<m b$. If $f$ is exponentially $m$-convex function, then the following inequalities hold:

$$
\begin{aligned}
& e^{f\left(\frac{a+m b}{2}\right)} G_{\sigma, \bar{\omega} 2^{\mu},\left(\frac{a+m b}{2}\right)^{+}}(m b ; p) \\
& \leq \frac{\left(\epsilon_{\mu, \sigma, q, \bar{\omega} 2^{\mu},\left(\frac{a+m b}{2}\right)^{+\rho, q, c}}^{+} e^{f}\right)(m b ; p)+m^{\sigma+1}\left(\epsilon_{\mu, \sigma, l, \bar{\omega}(2 m)^{\mu},\left(\frac{a+m b}{2 m}\right)^{\rho, r, q, c}}^{f}\right)\left(\frac{a}{m} ; p\right)}{2} \\
& \leq \frac{m^{\sigma+1}}{2(m b-a)}\left[\left(e^{f(a)}-m^{2} e^{f\left(\frac{a}{m^{2}}\right)}\right) G_{\sigma+1, \bar{\omega}(2 m)^{\mu},\left(\frac{a+m b}{2 m}\right)^{-}}\left(\frac{a}{m} ; p\right)\right. \\
& \left.+(m b-a)\left(e^{f(b)}+m e^{f\left(\frac{a}{m^{2}}\right)}\right) G_{\sigma, \bar{\omega}(2 m)^{\mu},\left(\frac{a+m b}{2 m}\right)^{-}}\left(\frac{a}{m} ; p\right)\right]
\end{aligned}
$$

where $m \in(0,1]$ and $\bar{\omega}$ is defined in $(2.2)$.

Proof. Putting $x=\frac{z}{2} a+m \frac{(2-z)}{2} b$ and $y=\frac{z}{2} b+\frac{(2-z)}{2} \frac{a}{m}$ in (2.3), we get

$$
2 e^{f\left(\frac{a+m b}{2}\right)} \leq e^{f\left(\frac{z}{2} a+m \frac{(2-z)}{2} b\right)}+m e^{f\left(\frac{z}{2} b+\frac{(2-z)}{2} \frac{a}{m}\right)} .
$$

Multiplying both sides of $(2.11)$ with $z^{\sigma-1} E_{\mu, \sigma, l}^{\rho, r, q}\left(\omega z^{\mu} ; p\right)$ and integrating over $[0,1]$, we have

$$
\begin{aligned}
& 2 e^{f\left(\frac{a+m b}{2}\right)} \int_{0}^{1} z^{\sigma-1} E_{\mu, \sigma, l}^{\rho, r, q, c}\left(\omega z^{\mu} ; p\right) d z \\
& \leq \int_{0}^{1} z^{\sigma-1} E_{\mu, \sigma, l}^{\rho, r, q, c}\left(\omega z^{\mu} ; p\right) e^{f\left(\frac{z}{2} a+\frac{(2-z)}{2} b\right)} d z \\
& +m \int_{0}^{1} z^{\sigma-1} E_{\mu, \sigma, l}^{\rho, r, q, c}\left(\omega z^{\mu} ; p\right) e^{f\left(\frac{z}{2} b+\frac{(2-z)}{2} \frac{a}{m}\right)} d z .
\end{aligned}
$$


Putting $u=\frac{z}{2} a+m \frac{(2-z)}{2} b$ and $v=\frac{z}{2} b+\frac{(2-z)}{2} \frac{a}{m}$ in (2.12), we get

$$
\begin{aligned}
& 2 e^{f\left(\frac{a+m b}{2}\right)} \int_{\frac{a+m b}{2}}^{m b}(m b-u)^{\sigma-1} E_{\mu, \sigma, l}^{\rho, r, q, c}\left(2^{\mu} \bar{\omega}(m b-u)^{\mu} ; p\right) d u \\
& \leq \int_{\frac{a+m b}{2}}^{m b}(m b-u)^{\sigma-1} E_{\mu, \sigma, l}^{\rho, r, q, c}\left(2^{\mu} \bar{\omega}(m b-u)^{\mu} ; p\right) e^{f(u)} d u \\
& +m^{\sigma+1} \int_{\frac{a}{m}}^{\frac{a+m b}{2 m}}\left(v-\frac{a}{m}\right)^{\sigma-1} E_{\mu, \sigma, l}^{\rho, r, q, c}\left((2 m)^{\mu} \bar{\omega}\left(v-\frac{a}{m}\right)^{\mu} ; p\right) e^{f(v)} d v .
\end{aligned}
$$

By using (1.7), (1.8) and (1.9), first inequality of (2.10) is achieved.

From exponentially $m$-convexity of $f$, we have

$$
\begin{aligned}
& e^{f\left(\frac{z}{2} a+m \frac{(2-z)}{2} b\right)}+m e^{f\left(\frac{z}{2} b+\frac{(2-z)}{2} \frac{a}{m}\right)} \\
& \leq \frac{z}{2} e^{f(a)}+m \frac{(2-z)}{2} e^{f(b)}+m\left(\frac{z}{2} e^{f(b)}+m \frac{(2-z)}{2} e^{f\left(\frac{a}{m^{2}}\right)}\right) \\
& =\frac{z}{2}\left(e^{f(a)}-m^{2} e^{f\left(\frac{a}{m^{2}}\right)}\right)+m\left(e^{f(b)}+m e^{f\left(\frac{a}{m^{2}}\right)}\right)
\end{aligned}
$$

Multiplying both sides of (2.13) with $z^{\sigma-1} E_{\mu, \sigma, l}^{\rho, r, q}\left(\omega z^{\mu} ; p\right)$ and integrating over $[0,1]$, we have

$$
\begin{aligned}
& \int_{0}^{1} z^{\sigma-1} E_{\mu, \sigma, l}^{\rho, r, q, c}\left(\omega z^{\mu} ; p\right) e^{f\left(\frac{z}{2} a+m \frac{(2-z)}{2} b\right)} d z \\
& +m \int_{0}^{1} z^{\sigma-1} E_{\mu, \sigma, l}^{\rho, r, q, c}\left(\omega z^{\mu} ; p\right) e^{f\left(\frac{z}{2} b+\frac{(2-z)}{2} \frac{a}{m}\right)} d z \\
& \leq \frac{1}{2}\left(e^{f(a)}-m^{2} e^{f\left(\frac{a}{m^{2}}\right)}\right) \int_{0}^{1} z^{\sigma} E_{\mu, \sigma, l}^{\rho, r, q, c}\left(\omega z^{\mu} ; p\right) d z \\
& +m\left(e^{f(b)}+m e^{f\left(\frac{a}{m^{2}}\right)}\right) \int_{0}^{1} z^{\sigma-1} E_{\mu, \sigma, l}^{\rho, r, q, c}\left(\omega z^{\mu} ; p\right) d z
\end{aligned}
$$

Putting $u=\frac{z}{2} a+m \frac{(2-z)}{2} b$ and $v=\frac{z}{2} b+\frac{(2-z)}{2} \frac{a}{m}$ in (2.14), we get

$$
\begin{aligned}
& \int_{\frac{a+m b}{2}}^{m b}(m b-u)^{\sigma-1} E_{\mu, \sigma, l}^{\rho, r, q, c}\left(2^{\mu} \bar{\omega}(m b-u)^{\mu} ; p\right) e^{f(u)} d u \\
& +m^{\sigma+1} \int_{\frac{a}{m}}^{\frac{a+m b}{2 m}}\left(v-\frac{a}{m}\right)^{\sigma-1} E_{\mu, \sigma, l}^{\rho, r, q, c}\left((2 m)^{\mu} \bar{\omega}\left(v-\frac{a}{m}\right)^{\mu} ; p\right) e^{f(v)} d v \\
& \leq \frac{m^{\sigma+1}}{m b-a}\left[\left(e^{f(a)}-m^{2} e^{f\left(\frac{a}{m^{2}}\right)}\right) \int_{\frac{a}{m}}^{\frac{a+m b}{2 m}}\left(v-\frac{a}{m}\right)^{\sigma} E_{\mu, \sigma, l}^{\rho, r, q, c}\left((2 m)^{\mu} \bar{\omega}\left(v-\frac{a}{m}\right)^{\mu} ; p\right) d v\right. \\
& \left.+(m b-a)\left(e^{f(b)}+m e^{f\left(\frac{a}{m^{2}}\right)}\right) \int_{\frac{a}{m}}^{\frac{a+m b}{2 m}}\left(v-\frac{a}{m}\right)^{\sigma-1} E_{\mu, \sigma, l}^{\rho, r, q, c}\left((2 m)^{\mu} \bar{\omega}\left(v-\frac{a}{m}\right)^{\mu} ; p\right) d v\right] .
\end{aligned}
$$

By using (1.7), (1.8) and (1.10), second inequality of (2.10) is achieved. 
Corollary 2.5. Suppose that assumptions of Theorem 2.4 hold and let $m=1$. Then following inequalities for exponentially convex function hold:

$$
\begin{aligned}
& e^{f\left(\frac{a+b}{2}\right)} G_{\sigma, \omega^{*} 2^{\mu},\left(\frac{a+b}{2}\right)^{+}}(b ; p) \\
& \leq \frac{\left(\begin{array}{c}
\epsilon_{\mu, r, q, c}^{\rho, \sigma, l, \omega^{*} 2^{\mu},\left(\frac{a+b}{2}\right)^{+}} \\
\leq
\end{array}\right)(b ; p)+\left(\epsilon_{\mu, \sigma, l, \omega^{*} 2^{\mu},\left(\frac{a+b}{2}\right)^{-}}^{\rho, r, q, c} e^{f}\right)(a ; p)}{2} \\
& \leq \frac{e^{f(a)}+e^{f(b)}}{2} G_{\sigma, \omega^{*} 2^{\mu},\left(\frac{a+b}{2}\right)^{-}}(a ; p)
\end{aligned}
$$

where $\omega^{*}$ is defined in (2.8).

Remark 2.6. If we take $\omega=p=0$ in (2.10), then [18, Theorem 3.3] is obtained.

In the following we give Fejér-Hadamard inequality for exponentially $m$-convex functions via generalized fractional integral operators.

Theorem 2.7. Let $\omega, \mu, \sigma, l, \rho, c \in \mathbb{C}, \Re(\mu), \Re(\sigma), \Re(l)>0, \Re(c)>\Re(\rho)>0$ with $p \geq 0$, $r>0$ and $0<q \leq r+\Re(\mu)$. Let $f:[a, m b] \subset \mathbb{R} \rightarrow \mathbb{R}$ be a function such that $f \in$ $L_{1}[a, m b]$ with $a<m b$. Also, let $g:[a, m b] \rightarrow \mathbb{R}$ be a function which is non-negative and integrable. If $f$ is exponentially $m$-convex function and $f(v)=f(a+m b-m v)$, then the following inequalities hold:

$$
\begin{aligned}
& e^{f\left(\frac{a+m b}{2}\right)}\left(\epsilon_{\mu, \sigma, l, \bar{\omega} m^{\mu}, b^{-}}^{\rho, r, q, c} e^{g}\right)\left(\frac{a}{m} ; p\right) \\
& \leq \frac{(1+m)\left(\epsilon_{\mu, \sigma, l, \bar{\omega} m^{\mu}, b^{-}}^{\rho, r, q, c} e^{f} e^{g}\right)\left(\frac{a}{m} ; p\right)}{2} \\
& \leq \frac{m}{2(m b-a)}\left[\left(e^{f(a)}-m^{2} e^{f\left(\frac{a}{m^{2}}\right)}\right)\left(\epsilon_{\mu, \sigma+1, l, \bar{\omega} m^{\mu}, b^{-}}^{\rho, r, q, c} e^{g}\right)\left(\frac{a}{m} ; p\right)\right. \\
& \left.+(m b-a)\left(e^{f(b)}+m e^{f\left(\frac{a}{m^{2}}\right)}\right)\left(\epsilon_{\mu, \sigma, l, \bar{\omega} m^{\mu}, b^{-}}^{\rho, r, q, c} e^{g}\right)\left(\frac{a}{m} ; p\right)\right]
\end{aligned}
$$

where $m \in(0,1]$ and $\bar{\omega}$ is defined in $(2.2)$.

Proof. Multiplying both sides of (2.4) with $z^{\sigma-1} E_{\mu, \sigma, l}^{\rho, r, q, c}\left(\omega z^{\mu} ; p\right) e^{g\left((1-z) \frac{a}{m}+z b\right)}$ and integrating over $[0,1]$, we have

$$
\begin{aligned}
& 2 e^{f\left(\frac{a+m b}{2}\right)} \int_{0}^{1} z^{\sigma-1} E_{\mu, \sigma, l}^{\rho, r, q, c}\left(\omega z^{\mu} ; p\right) e^{g\left((1-z) \frac{a}{m}+z b\right)} d z \\
& \leq \int_{0}^{1} z^{\sigma-1} E_{\mu, \sigma, l}^{\rho, r, q, c}\left(\omega z^{\mu} ; p\right) e^{f(z a+m(1-z) b)} e^{g\left((1-z) \frac{a}{m}+z b\right)} d z \\
& +m \int_{0}^{1} z^{\sigma-1} E_{\mu, \sigma, l}^{\rho, r, q, c}\left(\omega z^{\mu} ; p\right) e^{f\left((1-z) \frac{a}{m}+z b\right)} e^{g\left((1-z) \frac{a}{m}+z b\right)} d z .
\end{aligned}
$$


Putting $v=(1-z) \frac{a}{m}+z b$ in $(2.16)$, we get

$$
\begin{aligned}
& 2 e^{f\left(\frac{a+m b}{2}\right)} \int_{\frac{a}{m}}^{b}\left(v-\frac{a}{m}\right)^{\sigma-1} E_{\mu, \sigma, l}^{\rho, r, q, c}\left(m^{\mu} \bar{\omega}\left(v-\frac{a}{m}\right)^{\mu} ; p\right) e^{g(v)} d v \\
& \leq \int_{\frac{a}{m}}^{b}\left(v-\frac{a}{m}\right)^{\sigma-1} E_{\mu, \sigma, l}^{\rho, r, q, c}\left(m^{\mu} \bar{\omega}\left(v-\frac{a}{m}\right)^{\mu} ; p\right) e^{f(a+m b-m v)} e^{g(v)} d v \\
& +m \int_{\frac{a}{m}}^{b}\left(v-\frac{a}{m}\right)^{\sigma-1} E_{\mu, \sigma, l}^{\rho, r, q, c}\left(m^{\mu} \bar{\omega}\left(v-\frac{a}{m}\right)^{\mu} ; p\right) e^{f(v)} e^{g(v)} d v .
\end{aligned}
$$

By using (1.7), (1.8), (1.9) and given condition $f(v)=f(a+m b-m v)$, first inequality of (2.15) is achieved.

Now multiplying both sides of $(2.5)$ with $z^{\sigma-1} E_{\mu, \sigma, l}^{\rho, r, q, c}\left(\omega z^{\mu} ; p\right) e^{g\left((1-z) \frac{a}{m}+z b\right)}$ and integrating over $[0,1]$, we have

$$
\begin{aligned}
& \int_{0}^{1} z^{\sigma-1} E_{\mu, \sigma, l}^{\rho, r, q, c}\left(\omega z^{\mu} ; p\right) e^{f(z a+m(1-z) b)} e^{g\left((1-z) \frac{a}{m}+z b\right)} d z \\
& +m \int_{0}^{1} z^{\sigma-1} E_{\mu, \sigma, l}^{\rho, r, q, c}\left(\omega z^{\mu} ; p\right) e^{f\left((1-z) \frac{a}{m}+z b\right)} e^{g\left((1-z) \frac{a}{m}+z b\right)} d z \\
& \leq\left(e^{f(a)}-m^{2} e^{f\left(\frac{a}{m^{2}}\right)}\right) \int_{0}^{1} z^{\sigma} E_{\mu, \sigma, l}^{\rho, r, q, c}\left(\omega z^{\mu} ; p\right) e^{g\left((1-z) \frac{a}{m}+z b\right)} d z \\
& +m\left(e^{f(b)}+m e^{f\left(\frac{a}{m^{2}}\right)}\right) \int_{0}^{1} z^{\sigma-1} E_{\mu, \sigma, l}^{\rho, r, q, c}\left(\omega z^{\mu} ; p\right) e^{g\left((1-z) \frac{a}{m}+z b\right)} d z .
\end{aligned}
$$

From above second inequality of (2.15) is achieved.

Corollary 2.8. Suppose that assumptions of Theorem 2.7 hold and let $m=1$. Then following inequalities for exponentially convex function hold:

$$
\begin{aligned}
e^{f\left(\frac{a+b}{2}\right)}\left(\epsilon_{\mu, \sigma, l, \omega^{*}, b^{-}}^{\rho, r, q, c} e^{g}\right)(a ; p) & \leq\left(\epsilon_{\mu, \sigma, l, \omega^{*}, b^{-}}^{\rho, r, q, c} e^{f} e^{g}\right)(a ; p) \\
& \leq \frac{e^{f(a)}+e^{f(b)}}{2}\left(\epsilon_{\mu, \sigma, l, \omega^{*}, b^{-}}^{\rho, r, q, c} e^{g}\right)(a ; p)
\end{aligned}
$$

where $\omega^{*}$ is defined in (2.8).

Theorem 2.9. Let $\omega, \mu, \sigma, l, \rho, c \in \mathbb{C}, \Re(\mu), \Re(\sigma), \Re(l)>0, \Re(c)>\Re(\rho)>0$ with $p \geq 0, r>0$ and $0<q \leq r+\Re(\mu)$. Let $f, g:[a, m b] \subset \mathbb{R} \rightarrow \mathbb{R}$ be the functions such that $f, g \in L_{1}[a, m b]$ with $a<m b$. If $f$ and $g$ are exponentially $m$-convex functions, then the following inequality holds:

$$
\begin{aligned}
& \left(\epsilon_{\mu, \sigma, l, \bar{\omega}, m b^{-}}^{\rho, r, q, c} e^{f}\right)(a ; p)+\left(\epsilon_{\mu, \sigma, l, \bar{\omega}, a^{+}}^{\rho, r, q, c} e^{g}\right)(m b ; p) \\
& \leq \frac{1}{(m b-a)}\left[\left(e^{g(a)}+m e^{f(b)}\right) G_{\sigma+1, \bar{\omega}, a^{+}}(m b ; p)\right. \\
& \left.+\left(e^{f(a)}+m e^{g(b)}\right)\left\{(m b-a) G_{\sigma, \bar{\omega}, a^{+}}(m b ; p)-G_{\sigma+1, \bar{\omega}, a^{+}}(m b ; p)\right\}\right]
\end{aligned}
$$

where $m \in(0,1]$ and $\bar{\omega}$ is defined in $(2.2)$. 
Proof. Since $f$ and $g$ are exponentially $m$-convex, we have

$$
e^{f((1-z) a+m z b)}+e^{g(z a+m(1-z) b)} \leq(1-z)\left(e^{f(a)}+m e^{g(b)}\right)+z\left(e^{g(a)}+m e^{f(b)}\right) .
$$

Multiplying both sides of (2.18) with $z^{\sigma-1} E_{\mu, \sigma, l}^{\rho, r, q}\left(\omega z^{\mu} ; p\right)$ and integrating over $[0,1]$, we have

$$
\begin{aligned}
& \int_{0}^{1} z^{\sigma-1} E_{\mu, \sigma, l}^{\rho, r, q, c}\left(\omega z^{\mu} ; p\right) e^{f((1-z) a+m z b)} d z \\
& +\int_{0}^{1} z^{\sigma-1} E_{\mu, \sigma, l}^{\rho, r, q, c}\left(\omega z^{\mu} ; p\right) e^{g(z a+m(1-z) b)} d z \\
& \leq\left(e^{f(a)}+m e^{g(b)}\right) \int_{0}^{1}(1-z) z^{\sigma-1} E_{\mu, \sigma, l}^{\rho, r, q, c}\left(\omega z^{\mu} ; p\right) d z \\
& +\left(e^{g(a)}+m e^{f(b)}\right) \int_{0}^{1} z^{\sigma} E_{\mu, \sigma, l}^{\rho, r, q, c}\left(\omega z^{\mu} ; p\right) d z .
\end{aligned}
$$

Putting $u=(1-z) a+m z b$ and $v=z a+m(1-z) b$ in (2.19), then by using (1.7), (1.8) and (1.9), inequality (2.17) is achieved.

Corollary 2.10. Suppose that assumptions of Theorem 2.9 hold and let $m=1$. Then following inequality for exponentially convex function holds:

$$
\begin{aligned}
& \left(\epsilon_{\mu, \sigma, l, \omega^{*}, b^{-}}^{\rho, r, q, c} e^{f}\right)(a ; p)+\left(\epsilon_{\mu, \sigma, l, \omega^{*}, a^{+}}^{\rho, r, q} e^{g}\right)(b ; p) \\
& \leq \frac{1}{(b-a)}\left[\left(e^{g(a)}+e^{f(b)}\right) G_{\sigma+1, \omega^{*}, a^{+}}(b ; p)\right. \\
& \left.+\left(e^{f(a)}+e^{g(b)}\right)\left\{(b-a) G_{\sigma, \omega^{*}, a^{+}}(b ; p)-G_{\sigma+1, \omega^{*}, a^{+}}(b ; p)\right\}\right] .
\end{aligned}
$$

where $\omega^{*}$ is defined in (2.8).

Remark 2.11. If we take $\omega=p=0$ in (2.17), then [18, Theorem 3.2] is obtained.

Concluding remarks. The aim of this paper is to establish two versions of the fractional Hadamard inequalities for exponentially $m$-convex functions via generalized fractional integral operators. Further, a generalized version of the Hadamard inequality so called Fejér-Hadamard inequality is proved. The results of this paper are hold for various associated fractional integral operators.

Acknowledgment. The research work of the Ghulam Farid is supported by Higher Education Commission of Pakistan under NRPU 2016, Project No. 5421.

\section{References}

[1] Abbas, G., Farid, G., Hadamard and Fejér-Hadamard type inequalities for harmonically convex functions via generalized fractional integrals, J. Anal., 25(2017), no. 1, 107-119.

[2] Andrić, M., Farid, G., Mehmood, S., Pečarić, J., Pólya-Szegö and Chebyshev types inequalities via an extended generalized Mittag-Leffler function, Math. Ineq. Appl., 22(2019), no. 4, 1365-1377. 
[3] Andrić, M., Farid, G., Pečarić, J., A further extension of Mittag-Leffler function, Fract. Calc. Appl. Anal., 21(2018), no. 5, 1377-1395.

[4] Antczak, T., ( $p, r)$-invex sets and functions, J. Math. Anal. Appl., 263(2001), 355-379.

[5] Chen, F., On Hermite-Hadamard type inequalities for Riemann-Liouville fractional integrals via two kinds of convexity, Chin. J. Math., 2014(2014), pp 7.

[6] Chen, H., Katugampola, U.N., Hermite-Hadamard-Fejér type inequalities for generalized fractional integrals, J. Math. Anal. Appl., 446(2017), 1274-1291.

[7] Dragomir, S.S., Gomm, I., Some Hermite-Hadamard type inequalities for functions whose exponentials are convex, Stud. Univ. Babeş-Bolyai Math., 60(2015), no. 4, 527534.

[8] Farid, G., Hadamard and Fejér-Hadamard inequalities for generalized fractional integral involving special functions, Konuralp J. Math., 4(2016), no. 1, 108-113.

[9] Farid, G., A Treatment of the Hadamard inequality due to $m$-convexity via generalized fractional integral, J. Fract. Calc. Appl., 9(2018), no. 1, 8-14.

[10] Farid, G., Khan, K.A., Latif, N., Rehman, A.U., Mehmood, S., General fractional integral inequalities for convex and $m$-convex functions via an extended generalized MittagLeffler function, J. Inequal. Appl., 2018(2018), 243 pp.

[11] Farid, G., Rehman, A.U., Mehmood, S., Hadamard and Fejér-Hadamard type integral inequalities for harmonically convex functions via an extended generalized Mittag-Leffler function, J. Math. Comput. Sci., 8(2018), no. 5, 630-643.

[12] Farid, G., Rehman, A.U., Tariq, B., On Hadamard-type inequalities for $m$-convex functions via Riemann-Liouville fractional integrals, Stud. Univ. Babeş-Bolyai, Math., 62(2017), no. 2, 141-150.

[13] Fejér, L., Überdie Fourierreihen II, Math. Naturwiss Anz Ungar. Akad. Wiss., 24(1906), 369-390.

[14] Kang, S.M., Farid, G., Nazeer, W., Mehmood, S., $(h, m)$-convex functions and associated fractional Hadamard and Fejér-Hadamard inequalities via an extended generalized Mittag-Leffler function, J. Inequal. Appl., 2019(2019), 78 pp.

[15] Mittag-Leffler, G.M., Sur la nouvelle fonction $E_{\sigma}(x)$, Comptes Rendus Hebdomadaires des Séances de l' Académie des Sciences Paris, 137(1903), 554-558.

[16] Prabhakar, T.R., A singular integral equation with a generalized Mittag-Leffler function in the kernel, Yokohama Math. J., 19(1971), 7-15.

[17] Rahman, G., Baleanu, D., Qurashi, M.A., Purohit, S.D., Mubeen, S., Arshad, M., The extended Mittag-Leffler function via fractional calculus, J. Nonlinear Sci. Appl., 10(2017), 4244-4253.

[18] Rashid, S., Noor, M.A., Noor, K.I., Fractional exponentially $m$-convex functions and inequalities, Int. J. Anal. Appl., 17(2019), no. 3, 464-478.

[19] Rashid, S., Safdar, F., Akdemir, A.O., Noor, M.A., Noor, K.I., Some new fractional integral inequalities for exponentially $m$-convex functions via extended generalized MittagLeffler function, J. Inequal. Appl., 2019(2019), 299 pp.

[20] Salim, T.O., Faraj, A.W., A generalization of Mittag-Leffler function and integral operator associated with integral calculus, J. Frac. Calc. Appl., 3(2012), no. 5, 1-13.

[21] Sarikaya, M.Z., Set, E., Yaldiz, H., Basak, N., Hermite- Hadamard inequalities for fractional integrals and related fractional inequalities, J. Math. Comput. Model, 57(2013), no. 9, 2403-2407. 
[22] Sarikaya, M.Z., Yildirim, H., On Hermite-Hadamard type inequalities for RiemannLiouville fractional integrals, Miskolc Math. Notes, 17(2016), no. 2, 1049-1059.

[23] Shukla, A.K., Prajapati, J.C., On a generalization of Mittag-Leffler function and its properties, J. Math. Anal. Appl., 336(2007), 797-811.

[24] Srivastava, H.M., Tomovski, Z., Fractional calculus with an integral operator containing generalized Mittag-Leffler function in the kernal, Appl. Math. Comput., 211(2009), no. 1, 198-210.

Sajid Mehmood

Govt Boys Primary School Sherani, Hazro,

Attock, Pakistan

e-mail: smjg227@gmail.com

Ghulam Farid

COMSATS University Islamabad, Attock Campus,

Department of Mathematics,

Attock, Pakistan

e-mail: faridphdsms@hotmail.com, ghlmfarid@cuiatk.edu.pk 